\title{
Effect of Coulomb Interactions on Resolution in Ultra-fast Electron Microscopy
}

\author{
H. Rose and R. Spehr
}

Institute of Applied Physics, TU Darmstadt, Hochschulstrasse 6, 64289 Darmstadt, Germany

Pulsed electron beams are increasingly applied in dynamic electron microscopy for visualizing fast processes such as local changes of magnetization, phase transformations and chemical reactions. Photoemission electron microscopy with pulsed excitation sources like lasers or synchrotron radiation has been evolved as a standard method for observing dynamic processes on surfaces [1,2]. Recently it has been suggested to employ high-intensity ultra-short electron beams for recording single-shot diffraction patterns or images of radiation-sensitive biological objects [3].

Periodic processes can be observed with low current densities by means of stroboscopic techniques, which are mainly employed for investigating oscillating currents, voltages and magnetic domains in microelectronic devices. However, these techniques cannot be used for visualizing non-periodic processes or for obtaining a single-shot diffraction pattern or image, respectively. In order to achieve high-spatial resolution, the pulse must contain as many electrons as possible. However, due to the repulsive Coulomb interaction between electrons, it is not possible to confine a very large number of electrons in a very small volume. Moreover, these interactions broaden the energy width of the image-forming beam and change the direction of flight of the electrons. As a result, the image spot will be broadened even if chromatic aberration has been corrected [4]. This loss of resolution will also arise for the diffraction pattern.

The mean space charge acts like a change of the focal length and of the aberrations of the electron lenses. Therefore, this stationary effect can be compensated in an aberration-corrected microscope. Unfortunately, this correction is not possible for the effects resulting from the stochastic interactions. Ray deflections arising within the lenses from stochastic interactions are most deleterious, because even small angular deviations may result in appreciable displacements at the image plane owing to the large distance between the lenses and the image plane. This deleterious effect also prevails for a lens-less system, where it reduces the resolution of the diffraction pattern. The broadening of the diffraction or image spots resulting from stochastic interactions is inversely proportional to $U^{* 3 / 4}$. Here $U^{*}=U\left(1+e U / 2 E_{e}\right)$ defines the relativistic modified acceleration voltage and $E_{e} \approx 0.51 \mathrm{MeV}$ is the rest energy of the electron. Hence, the resolution increases significantly by going to higher voltages if the geometry of the system remains fixed. However, this condition cannot be met for an imaging system because the focal length of the magnetic lenses increases in proportion to $U^{*}$ whenever their magnetic field has reached its maximum. Therefore, going to high voltages will only improve the resolution limited by stochastic interactions if superconducting lenses with short focal lengths are employed. Projector systems composed of quadrupoles are another alternative because quadrupoles are strong-focusing elements and can form astigmatic intermediate images of the source, thus reducing the current density and the resulting stochastic ray deflections.

Single elastic scattering prevails in amorphous objects whose thickness $t$ is smaller than the elastic mean free path length.. For simplicity we assume that the object is composed of atoms with the same atomic number Z. In this case the relation $l_{e l}=1 / n_{A} \sigma_{e l}$ holds. Here $n_{A}$ is the atom density of the object and $\sigma_{e l} \approx\left(E_{e} / e U^{*}\right)\left(1+e U / E_{e}\right)^{2} Z^{4 / 3} \mathrm{pm}^{2}$ the elastic scattering cross-section of the atom. This cross-section 
is inversely proportional to $U$ in the non-relativistic regime and adopts the constant value $2 Z^{4 / 3} \mathrm{pm}^{2}$ in the relativistic case $U>>2 E_{e} \approx 1 \mathrm{MeV}$. For imaging thin $\left(t<l_{e l}\right)$ amorphous objects, it is advantageous to operate at lower voltages due to the larger fraction of scattered electrons. In addition, one can reduce the number of interactions in the regime between object and image by removing the non-scattered electrons in the back-focal plane of the objective lens by means of a beam stop. Two modes of dark-field imaging exist, as depicted in Fig.1. The first mode uses parallel illumination and a central beam stop, the other hollow cone illumination and a circular aperture. Compared to parallel illumination this mode reduces the effect of the Coulomb interactions due to the large angles of illumination which shorten the regions of high current density.

Instead of employing an annular aperture and a standard cathode for producing hollow cone illumination, it may be possible to develop a pulsed high-intensity annular cathode. We achieve hollow-cone Koehler illumination by imaging this cathode into the back-focal plane of the objective lens. In order to minimize the Coulomb interactions and to utilize as many scattered electrons as possible large usable angles are required for the illumination hollow cone and the objective aperture. Such angles can only be realized by correcting the resolution-limiting aberrations.

a)

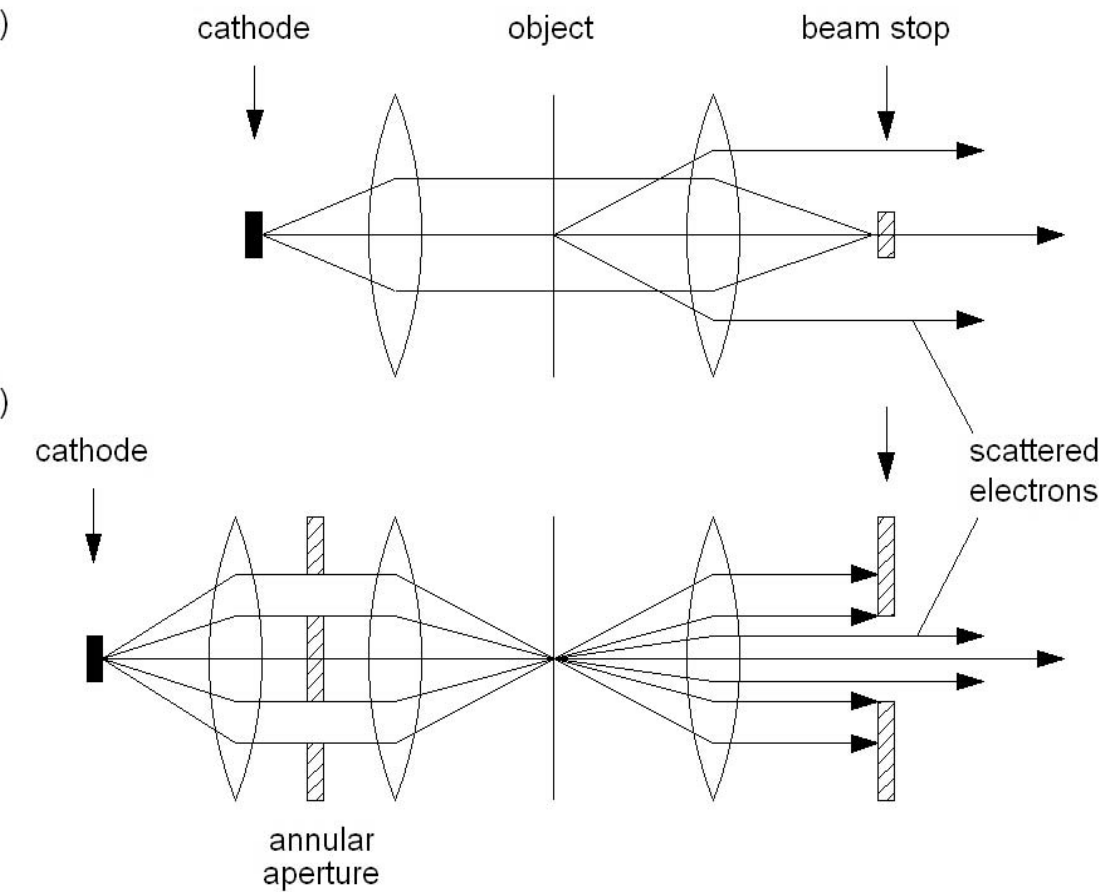

b)

Fig.1. Schematic arrangement of apertures and lenses for (a) central beam stop and (b) hollow-cone darkfield imaging

\section{References}

[1] O. Bostanjoglo and M. Weingaertner, Rev. Sci. Instr. 68 (1997) 2456.

[2] A. Krasyuk et al., Appl. Phys. A 76 (2003) 863.

[3] W.King et al. J. Appl. Phys. 97 (2005) 111101.

[4] A. Weidenhausen, R. Spehr, H. Rose, Optik 69 (1985) 126. 